\title{
IBS, aDIS/BMS, Astec, RDS, ZDV, Konsortialmodell, ... ???
}

\section{Das zukünftige Bibliothekssystem für die WLB}

Eine große und leistungsfähige Bibliothek benötigt ein stabiles und verlässliches IT-Rückgrat, um benutzer- und mitarbeiterbezogen effektiv und zuverlässig arbeiten zu können. Die bibliothekarischen Tätigkeiten und Geschäftsgänge werden dabei durch Fachanwendungen unterstützt, und die damit implementierten Workflows umspannen zumeist mehrere Bereiche einer Bibliothek.

Die EDV-Landschaft der meisten Bibliotheken, so auch der Württembergischen Landesbibliothek (WLB), ist historisch gewachsen und durch mehrere Randbedingungen geprägt worden, wie sie ähnlich auch in anderen wissenschaftlichen Bibliotheken in Baden-Württemberg vorliegen. Mit der Einführung der Olaf-Systeme zur Realisierung einer automatisierten Ausleihe hat im Mai 1984 erstmals die Datenverarbeitung Einzug in die WLB gehalten. Durch die Jahr-2000-Problematik musste damals dieses in Baden-Württemberg weit verbreitete System dringend abgelöst werden. Das Ablösesystem sollte dabei nicht nur die Ausleihfunktionen realisieren, sondern auch andere Aufgabenbereiche in einer Bibliothek unterstützen. Ziel war es, landesweit ein integriertes Bibliothekssystem einzuführen. Integriertes Bibliothekssystem (= IBS) bedeutet dabei, dass die Bereiche Ausleihe, Fernleihe, Erwerbung, Zeitschriften, Lizenzen und OPAC durch ein System verwaltet werden. Aus verschiedenen Gründen war damals dieses Vorhaben jedoch nicht für die großen Bibliotheken im Lande zu realisieren. Die WLB hat daraufhin im Jahr 2000 das Ausleihsystem Bibdia eingeführt, das sich bis heute zuverlässig im Einsatz befindet. Damit wurde jedoch nur der Ist-Zustand fortgeschrieben, und es konnte keine qualitative Verbesserung durch Integration erreicht werden. Dringender Handlungsbedarf zur IT-Unterstützung der internen Abläufe zeichnete sich auch in dem Bereich der Medienbearbeitung/ Erwerbung ab. Landesweit kam es zu einer Ausschreibung eines Erwerbungssystems. Die Firma LIB-IT bekam seinerseits mit dem Produkt LIBERO den Zuschlag. Die WLB ging mit diesem System 2005 in Produktion. Als Benutzer-OPAC kam in der WLB seit 1996 der sogenannte OLIX-OPAC zum Einsatz. Dieser von der UB Stuttgart, UB Hohenheim und der WLB gemeinsam im Rechenzentrum der Universität Stuttgart betriebene OPAC musste ebenfalls aus technischen Gründen dringend abgelöst werden. Die WLB entschied sich für die Karlsruher Entwicklung XOPAC, der seit 2008 auf eigenen Servern betrieben wird.

Der Ist-Zustand der Bibliotheks-IT in der WLB ist also gekennzeichnet durch eine Vielzahl von getrennten Bibliothekssystemen, die zunächst untereinander nicht kommunizieren. Die Aufgabe der IT-Abteilung bestand nun darin, diese isolierten Systeme über deren vorhandene Schnittstellen miteinander in Beziehung zu setzen, um so zumindest ein lose gekoppeltes, einfach integriertes Gesamtbibliothekssystem zu realisieren. Beispielsweise muss natürlich der OPAC an das Ausleihsystem angeschlossen sein oder die elektronische Fernleihe an das Bibdia-System. Neben diesem "eigenen" System zeichnete sich in den letzten Jahren auch verstärkt eine bibliotheksübergreifende Vernetzung von Diensten ab. Die elektronische Fernleihe, EZB, DBIS, eBooks, BibTip, digitale Objekte mit deren Metadaten usw. müssen in das Gesamtsystem integriert werden. Die noch vereinfachte Darstellung in Abbildung 1 muss dabei nicht im Detail verstanden oder nachvollzogen werden, sondern soll einen Eindruck davon wiedergeben, welche Systeme wo in welcher Beziehung stehen, und welche Komplexität dem Gesamtsystem innewohnt. Ein solch lose gekoppeltes, teil-integriertes System hat Vor- und Nachteile. Alle Teilsysteme arbeiten sehr stabil und performant. Sie sind für den Massendurchsatz geeignet und im Laufe der Zeit für die Geschäftsgänge und die Anforderungen der WLB optimiert und erweitert worden. Auf der anderen Seite ist es für das IT-Personal ein zeitaufwändiges Unterfangen, die handgemachte Integra- 


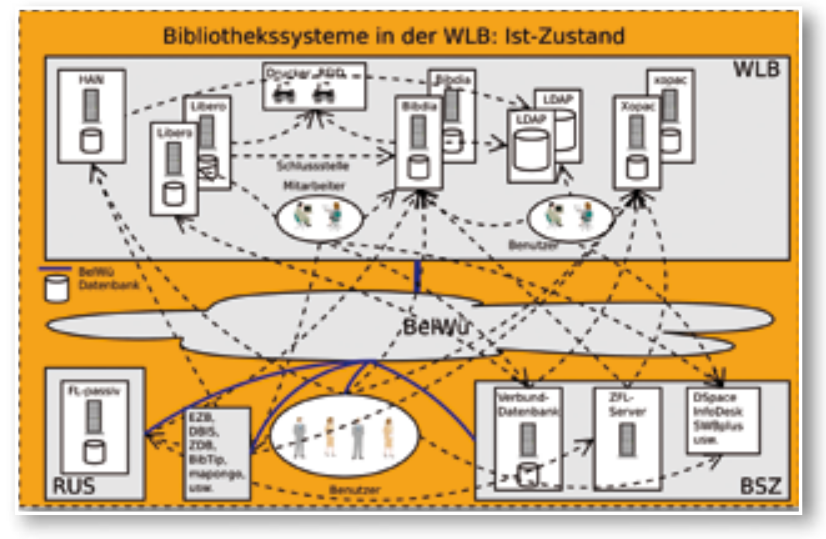

Abbildung 1: Bibliothekssystem in der WLB: Ist-Zustand

tion datenmäßig konsistent (jedes Teilsystem hat seine eigene Datenbank) und verfügbar zu halten, sowie weitere, neue Dienste anzudocken.

Um die IT-Infrastuktur in den wissenschaftlichen Bibliotheken in Baden-Württemberg zu verbessern, zu konsolidieren, bzw. um abgekündigte Systeme zu ersetzen ${ }^{1}$, wurde seit ca. 2008 zur Vorbereitung einer europaweiten Ausschreibung ${ }^{2}$ für ein integriertes Bibliothekssystem ein umfangreiches Pflichtenheft erstellt. An diesem Pflichtenheft arbeiteten aus den beteiligten Bibliotheken jeweils die Spezialisten für Benutzung/Ausleihe, Erwerbung/Medienbearbeitung und IT mit. Auch in der WLB wurden in diesem Zusammenhang umfangreiche Fragebögen bearbeitet, Checklisten erstellt und Anforderungen bezüglich deren Bedeutung für die WLB gewichtet und bewertet. Im Rahmen dieser Ausschreibung erhielt am 04.12.2009 die Firma aStec $\mathrm{GmbH}^{3}$ mit dem Bibliotheksmanagementsystem aDIS/BMS den Zuschlag für eine Landeslizenz Baden-Württemberg ${ }^{4}$.

Ein gemeinsames integriertes Bibliothekssystem hat nun eine Reihe von Vorteilen. Die Zusammenarbeit und die gegenseitige Unterstützung kann dank gleicher Systemumgebungen noch besser als bisher erfolgen. Ferner bewirkt die Reduzierung der Schnittstellenvielfalt eine Verbesserung der Kooperation mit externen Partnern, da Anpassungen nicht $\mathrm{n}$-fach implementiert werden müssen. Authentifizierungssysteme wie z.B. LDAP oder Shibboleth können für alle teilnehmenden Bibliotheken verwendet werden.

Die Einführung eines integrierten Bibliothekssystems in dieser Größenordnung (ca. 48 Hochschulbibliotheken, die vom BSZ betreut werden, sowie 7 große wissenschaftliche Bibliotheken sind beteiligt) kann nun nicht einfach so "nebenbei" realisiert werden, sondern muss projektmäßig organisiert sein. In Zusammenarbeit mit den „IBSDirektoren" wurde eine Organisationsstruktur für das IBS-Projekt festgelegt, ein Migrationszeitplan verabschiedet und ein Betriebsmodell erarbeitet.

Das IBS-Projektmanagment ist am kiz Ulm angesiedelt, die Projektleitung hat Herr Prof. Grossmann übernommen. Die IBS-Direktoren stimmen sich dabei mit der Projektleitung regelmäßig ab und treffen strategische Entscheidungen. Ferner unterstützt die Arbeitsgruppe IBS, in der die großen Bibliotheken jeweils mit zwei Vertretern mitarbeiten (eine bibliothekarische und eine ITFachkraft), das IBS-Projekt, indem auf Arbeitsebene Fragestellungen diskutiert und Entscheidungen getroffen werden. Bei Bedarf können, in Abstimmung mit der Projektleitung, Unter-AGs (z.B. für OPAC-, Statistikfragen usw.) gebildet werden.

Das Betriebsmodell des IBS-Projekts legt fest, wie, an welchen Standorten, auf welcher Hardware, mit welcher Netzanbindung, Verfügbarkeit, Reaktionszeit, mit welchen Rechten und welcher Aufgabenverteilung das Bibliothekssystem konkret betrieben werden soll. Für das IBS-Projekt wurde ein zentrales Betriebsmodell festgelegt und das ZDV 5 der Universität Tübingen mit dem Hosting und Betrieb der Anwendung beauftragt. Im ZDV wurden an zwei räumlich getrennten Standorten redundante Server- und Netzwerkinfrastrukturen aufgebaut, auf denen die aDIS/BMS-Anwendung läuft. Da mit virtuellen Instanzen gearbeitet wird,

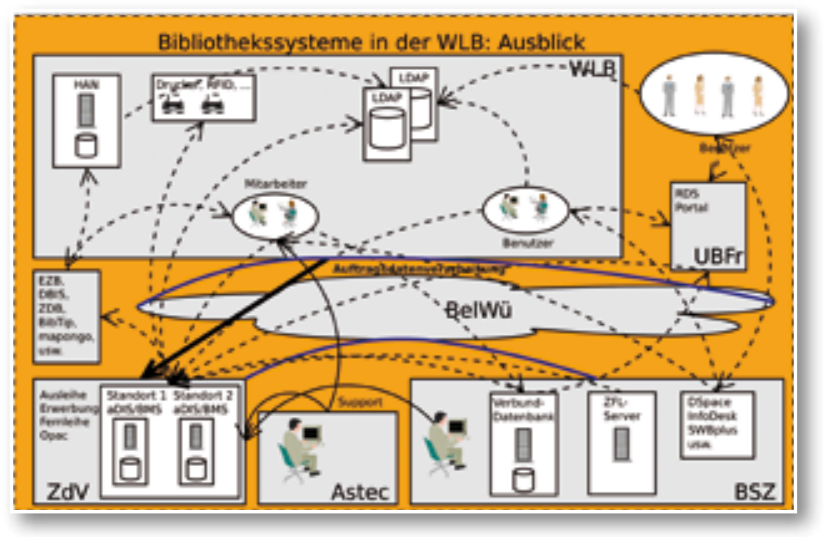

Abbildung 2: Bibliothekssystem in der WLB: Ausblick

(1) z.B. Horizon, Bibdia Windows-Client, usw.

(2) Beteiligte Bibliotheken: BLB, BSZ (mit ca. 48 Hochschulbibliotheken), UB Freiburg, UB Hohenheim (KIM), UB Stuttgart, UB Tübingen, kiz UIm, WLB

(3) www.astec.de

(4) Diese Landeslizenz umfasst die Bibliotheken im Geschäftsbereich des MWK.

(5) ZDV: Zentrum für Datenverarbeitung 
kann die Hardwareinfrastruktur sehr effektiv genutzt werden. Das ZDV sorgt für einen sicheren Betrieb, spielt Patches und Updates ein und stellt die Verfügbarkeit sicher. Dabei arbeitet das ZDV sehr intensiv mit der Firma Astec und ggf. weiteren Experten zusammen.

Für das Hosting und den Betrieb eines solch großen Projektes liegen bisher, sowohl von Seiten der Firma Astec als auch von Seiten des ZDVs, noch wenig Erfahrungen bzgl. Ressourcenbedarf ${ }^{6}$ und Skalierbarkeit vor. Das gehostete System ist immerhin das Herzstück aller teilnehmenden Bibliotheken, und Enpässe wirken sich sofort auf Mitarbeiter- und Benutzerfunktionen aus. Um eine gute Antwortzeit zu gewährleisten, wird daher die Hardwareausstattung Zug um Zug mit fortschreitenden Migrationen und zunehmender Auslastung ausgebaut.

In der Abbildung 2 wird das neue Betriebsmodell aus Sicht der WLB skizziert. Es fällt Folgendes auf: Im alten, lose gekoppelten Betriebsmodell können Teilsysteme ausfallen, und dennoch sind davon nicht betroffene Teilfunktionen vor Ort weiterhin nutzbar (z.B. die Libero-Erwerbung bei Ausfall der Ausleihe). Mit dem neuen Betriebsmodell spielt die Qualität des Hostings und der Netzanbindung eine erheblich größere Rolle. Ohne funktionsfähige Internetanbindung wird kein Betrieb mehr möglich sein. Im Zuge des IBS-Projektes wird daher die WLB eine schnellere (1 GBit/s) und vor allem über getrennte Trassen geführte, redundante Anbindung an das BelWü-Netz ${ }^{7}$ bekommen.

\section{aDIS/BMS wird für das Konsortium in mehreren} Instanzen betrieben. Das BSZ bekommt eine eigene Instanz und realisiert darin alle Hochschulbibliotheken als Mandanten ${ }^{8}$. Die großen wissenschaftlichen Bibliotheken - so auch die WLB - bekommen jeweils eine eigene aDIS/BMS-Instanz und ein Testsystem. Trotz zentralem Betriebsmodell können daher die Bibliotheken sehr eigenständige, voneinander unabhängige Konfigurationen vornehmen.

(6) Plattenplatz, I/O, RAM, SSD,

(7) Be/Wü: Ist das Datennetz der wissenschaftlichen Einrichtungen des Landes Baden Württemberg. Für die WLB ist das BelWü der Internetprovider.

(8) aDIS/BMS ist voll mandantenfähig. Diese Eigenschaft spielt für die WLB keine große Rolle. Die Universitäten können aber z.B. die Institute als Mandanten realisieren.

(9) TOM: Technische und organisatorische Maßnahmen, die festlegen, dass die Sicherheits- und Schutzanforderungen nach BDSG bei der Verarbeitung personenbezogener Daten eingehalten werden.

(10) Davon konnten wir uns in der Stadtbibliothek München überzeugen.
Das zentrale Betriebsmodell bedeutet nun aber auch, dass zum Teil personenbezogene Daten nicht mehr in der eigenen Einrichtung verarbeitet werden, sondern in einem externen Rechenzentrum. Das ist ein typischer Fall von Auftragsdatenverarbeitung und bedeutet, dass neben allen technischen Migrationsarbeiten auch ein entsprechendes Datensicherheits- und Datenschutzkonzept zwischen den jeweils relevanten Partnern erarbeitet und realisiert werden muss. Es ist also nicht nur eine Dienstvereinbarung zwischen dem örtlichen Personalrat und der Bibliotheksleitung bzw. die Erstellung eines Verfahrensverzeichnisses notwendig, sondern es ist zudem die Erstellung einer großen Anzahl von Dokumenten sowie die Durchführung von Prüfungen und Begehungen zwischen den Projektbeteiligten (ZDV, kiz, Astec, BSZ, Zendas, WLB usw.) erforderlich, um die Anwendung datenschutzrechtlich und datensicher korrekt betreiben zu können. So muss beispielsweise ein Datenschutz- , Sicherheits- und Löschkonzept erstellt werden, es müssen Zuverlässigkeitsprüfungen erfolgen und eine Beschreibung der $\mathrm{TOMs}^{9}$.

Für die WLB wird die Migration Ende 2012 konkret werden. Alle Migrationsaktivitäten werden in einem eigenen WLB-Wiki dokumentiert, auf das die an der Migration aktiv beteiligten Mitarbeiter schreibenden Zugriff erhalten. In einer ersten Phase wird es ein Kick-Off-Gespräch geben und das WLB-Testsystem eingerichtet werden. Weiter werden die Altdaten analysiert, eine Testmigration durchgeführt und die Tester geschult. Für diese Aufgaben sind ungefähr drei Monate vorgesehen. In der zweiten Migrationsphase wird dann die endgültige Migration vorbereitet und die Konfiguration des Produktionssystems nach Vorgaben der WLB in Zusammenarbeit mit der Firma Astec erfolgen. In dieser Phase werden auch die Mitarbeiter für die jeweils relevanten Module nach WLB-Gesichtspunkten geschult. Die altbekannte Software für Bibdia und Libero wird "abgeschaltet" und durch die aDIS/BMS-Software, die es als Windows- oder als Browser-Version gibt, ersetzt. Bei der Ausschreibung des Bibliothekssystems war ein wichtiges Softwarekriterium die Tauglichkeit für den Massenbetrieb ${ }^{10}$. So können mit aDIS/BMS viele Thekentätigkeiten ohne Maus, nur mit Tastatur und Shortcuts, effizient durchgeführt werden. Zur Katalogisierung wird weiterhin die WinIBW zum Einsatz kommen. 
Eine wesentliche Aufgabe der Migration wird die Zusammenführung der vorhandenen Altdaten und ihre Aufbereitung für das Zielsystem sein. Im Altsystem liegen die relevanten Daten in getrennten Datenbanken. Titel- und Lokaldaten werden vom Verbund geliefert. Aus Bibdia müssen die Ausleih-, Bewegungs-, Benutzer- und Mediendaten exportiert werden. Die Liberodatenbank enthält die seit 2005 angelegten Erwerbungsdaten. Aus dem Erwerbungssystem sollen beispielsweise die Lieferantensätze (über 10.000 Stück), die offenen Bestellungen, die Zeitschriftenabonnements, die Rechnungsdaten und die Exemplarsätze in das neue System übernommen werden. Diese LiberoExemplarsätze müssen dann mit den zusätzlichen Exemplarinformationen aus dem Bibdia-System und den SWB-Lokalsätzen zu aDIS-Exemplaren zusammengefasst werden. Das sind umfangreiche, langandauernde Export- und Importroutinen. Daher wird die Migration kaum ohne Schließtage durchzuführen sein. Mit der Migration muss ferner auch die Ansteuerung der Peripheriegeräte wie Magazindrucker, ggf. Ausweisdrucker, RFID-Hardware, Kassenautomat usw. auf das aDIS/BMS-System umgestellt werden.

An die vorhandenen Bibliothekssysteme sind im Laufe der Zeit eine Menge von abgeleiteten Diensten „angehängt" worden: z.B. umfangreiche Statistikauswertungen, sortierter Magazindruck, Etikettendruck, systematische Standortkataloge, Benutzerverwaltungsfunktionen, Benutzerauthentifizierungen via LDAP, Fernleihfunktionen usw. Mit der Migration müssen die Schnittstellen dieser Sekundärdienste so angepasst werden, dass diese zukünftig mit aDIS/BMS zusammenarbeiten können.

In aDIS/BMS ist die RFID-Unterstützung bereits weitgehend integriert. Die WLB plant daher nach der Migration in einem zweiten Schritt im Selbstabholerbereich RFID-Selbstverbuchungsstationen aufzustellen und zur Buchsicherung entsprechende Sicherungsgates zu installieren.

Nach erfolgter Migration wird auch der XOPAC abgeschaltet und die dort integrierten Dienste (z.B. mapongo, BibTip, PaperC usw. ) in den neuen aDIS-OPAC integriert werden.
Im Zeitraum zwischen der Erstellung des IBSPflichtenheftes und der Einführung des IBSSystems hat es im Umfeld der OPAC- und RDSProdukte ${ }^{11}$ wesentliche Weiterentwicklungen gegeben. Es geht nicht mehr nur um "einfache" OPAC-Funktionen, sondern um das Auffinden von Ressourcen und um den schnellen, direkten Zugriff auf das Buch, den Zeitschriftenartikel, einen Scan oder ein sonstiges, lizenziertes, digitales Objekt. Grundlage für solche RDS-Systeme sind große Indexe, die kommerziell erworben werden können. Solche RDS-Systeme können einen lokalen OPAC ersetzen, wenn eine Verknüpfung vom RDSSystem zum Lokalsystem realisiert werden kann. Die UB Freiburg wurde beauftragt, für das IBSProjekt ein solches RDS-System zu entwickeln, und Astec wird die entsprechenden Schnittstellen bereitstellen. Diese Lösung wird auf den Komponenten VuFind, ReDI-Linkresolver und einem Proxy zur standardisierten Anbindung von externen Indexen realisiert werden.

Das nächste Jahr wird für die WLB - nicht nur bibliothekstechnisch gesehen - spannend werden. Die meisten Bibliotheksbereiche werden von der Migration auf das neue Bibliothekssystem betroffen sein. Das erfordert für alle Offenheit für neue Konzepte und neue Lösungsansätze sowie Lernbereitschaft, sich in die neue Software einzuarbeiten. Vermutlich wird nicht alles gleich so rund und effektiv laufen wie im Zoo der Altsysteme. Allerdings zeichnen sich die Altsysteme auch dadurch aus, dass in vielen Jahren die Konfiguration optimiert und passende Erweiterungen programmiert werden konnten. Auch das neue System muss erst richtig durchschaut, verstanden und verinnerlicht werden, um es gut an die WLB-spezifische (Pflicht-) Bibliothekssituation anpassen zu können. Das neue System soll schließlich einen qualitativen Mehrwert generieren.

Für diese Mammutaufgabe wünsche ich uns allen viel Ausdauer, Experimentierfreude, einen langen Atem, eine gute Kommunikation sowie konstruktive Gedanken und Beiträge, damit wir unsere Bibliothek mit aDIS/BMS fit für die Zukunft halten.

Stephan Abele 